\title{
Computional algorithm for lifetime exposure to antimicrobials in pigs using register
} data the LEA algorithm

Birkegård, Anna Camilla; Dalhoff Andersen, Vibe; Hisham Beshara Halasa, Tariq; Jensen, Vibeke Frøkjær; Toft, Nils; Vigre, Håkan

Published in:

Preventive Veterinary Medicine

Link to article, DOI:

10.1016/j.prevetmed.2017.08.008

Publication date:

2017

Document Version

Peer reviewed version

Link back to DTU Orbit

Citation (APA):

Birkegård, A. C., Dalhoff Andersen, V., Hisham Beshara Halasa, T., Jensen, V. F., Toft, N., \& Vigre, H. (2017). Computional algorithm for lifetime exposure to antimicrobials in pigs using register data the LEA algorithm. Preventive Veterinary Medicine, 146, 173-180. https://doi.org/10.1016/j.prevetmed.2017.08.008

\section{General rights}

Copyright and moral rights for the publications made accessible in the public portal are retained by the authors and/or other copyright owners and it is a condition of accessing publications that users recognise and abide by the legal requirements associated with these rights.

- Users may download and print one copy of any publication from the public portal for the purpose of private study or research.

- You may not further distribute the material or use it for any profit-making activity or commercial gain

- You may freely distribute the URL identifying the publication in the public portal 


\section{Accepted Manuscript}

Title: Computional algorithm for lifetime exposure to

antimicrobials in pigs using register data - the LEA algorithm

Authors: Anna Camilla Birkegård, Vibe Dalhoff Andersen,

Tariq Halasa, Vibeke Frøkjær Jensen, Nils Toft, Håkan Vigre

PII: $\quad$ S0167-5877(16)30388-9

DOI: $\quad$ http://dx.doi.org/doi:10.1016/j.prevetmed.2017.08.008

Reference: $\quad$ PREVET 4302

To appear in: $\quad$ PREVET

Received date: $\quad 21-9-2016$

Revised date: $\quad$ 7-8-2017

Accepted date: $\quad 10-8-2017$

Please cite this article as: Birkegård, Anna Camilla, Andersen, Vibe Dalhoff, Halasa, Tariq, Jensen, Vibeke Frøkjær, Toft, Nils, Vigre, Håkan, Computional algorithm for lifetime exposure to antimicrobials in pigs using register data - the LEA algorithm.Preventive Veterinary Medicine http://dx.doi.org/10.1016/j.prevetmed.2017.08.008

This is a PDF file of an unedited manuscript that has been accepted for publication. As a service to our customers we are providing this early version of the manuscript. The manuscript will undergo copyediting, typesetting, and review of the resulting proof before it is published in its final form. Please note that during the production process errors may be discovered which could affect the content, and all legal disclaimers that apply to the journal pertain. 
Title page

Title: Computional algorithm for lifetime exposure to antimicrobials in pigs using register data - the LEA algorithm.

Authors: Anna Camilla Birkegård ${ }^{1 *}$, Vibe Dalhoff Andersen², Tariq Halasa ${ }^{1}$, Vibeke Frøkjær Jensen $^{1}$, Nils Toft ${ }^{1}$, Håkan Vigre ${ }^{2}$

1: National Veterinary Institute, Technical University of Denmark, Kemitorvet, 2800 Kgs. Lyngby, Denmark

2: National Food Institute, Technical University of Denmark, Kemitorvet, 2800 Kgs. Lyngby, Denmark

*corresponding author, acbir@vet.dtu.dk 


\begin{abstract}
Accurate and detailed data on antimicrobial exposure in pig production are essential when studying the association between antimicrobial exposure and antimicrobial resistance. Due to difficulties in obtaining primary data on antimicrobial exposure in a large number of farms, there is a need for a robust and valid method to estimate the exposure using register data.
\end{abstract}

An approach that estimates the antimicrobial exposure in every rearing period during the lifetime of a pig using register data was developed into a computational algorithm. In this approach data from national registers on antimicrobial purchases, movements of pigs and farm demographics registered at farm level are used. The algorithm traces batches of pigs retrospectively from slaughter to the farm(s) that housed the pigs during their finisher, weaner, and piglet period. Subsequently, the algorithm estimates the antimicrobial exposure as the number of Animal Defined Daily Doses for treatment of one $\mathrm{kg}$ pig in each of the rearing periods. Thus, the antimicrobial purchase data at farm level are translated into antimicrobial exposure estimates at batch level. A batch of pigs is defined here as pigs sent to slaughter at the same day from the same farm.

In this study we present, validate, and optimise a computational algorithm that calculate the lifetime exposure of antimicrobials for slaughter pigs. The algorithm was evaluated by comparing the computed estimates to data on antimicrobial usage from farm records in 15 farm units. We found a good positive correlation between the two estimates.

The algorithm was run for Danish slaughter pigs sent to slaughter in January to March 2015 from farms with more than 200 finishers to estimate the proportion of farms that it was applicable for. In the final process, the algorithm was successfully run for batches of pigs originating from 3,026 farms with finisher units (77 \% of the initial population). This number can be increased if more accurate register data can be obtained. 
The algorithm provides a systematic and repeatable approach to estimating the antimicrobial exposure throughout the rearing period, independent of rearing site for finisher batches, as a lifetime exposure measurement.

Abbreviations:

LEA: Lifetime exposure to antimicrobials

CHR: Central Husbandry Register

PMD: Database of Pig Movements

$\mathrm{ADD}_{\mathrm{kg}}$ : Animal Defined Daily Dose for treatment of one kg pig

Keywords: antimicrobial data, pig population, algorithm, movement pattern 


\section{Introduction}

In recent decades, authorities have enforced regulatory initiatives to regulate the usage of antimicrobials in an attempt to reduce antimicrobial resistance (AMR) in livestock animals. However, there is a general need for a quantitative assessment of the relationship between AMR and antimicrobial exposure in livestock animals. In studies of this relationship, reliable estimates of antimicrobial exposure in batches of livestock animals during different rearing periods are needed. Here, we define a batch of animals as animals sent to slaughter on the same day from the same farm. In modern conventional pig production, the time from birth to slaughter is approximately six months. Therefore, we assume that the antimicrobial exposure during the entire lifetime of pigs influences the levels of AMR found at slaughter. Previous studies performed in pig farms have shown that AMR at one time point was associated with antimicrobial exposure in previous rearing periods (Dunlop et al., 1998; Rosengren et al., 2007; Varga et al., 2009). Furthermore, in population studies estimates of antimicrobial exposure must reflect the actual use in batches during the lifetime of the pigs. This can be difficult to obtain when using farm level register data because pigs are often moved from the farm, where they were born.

In this study, we present a computational algorithm that use data from registers to translate antimicrobial purchase data on farm level to antimicrobial exposure on batch level. The antimicrobial exposure is calculated for three rearing periods (piglet, weaner, and finisher period) and as a total estimate of the amount of antimicrobial exposure. We call this the LEA approach. The concept of the LEA approach was presented by Andersen et al. (2017), who showed that the LEA estimate explained more of the variation in the AMR levels in finishers than estimates of the antimicrobial usage in finisher units only or at the farms in general did. The principle of the LEA approach presented by Andersen et al (2017) was a database estimation of the amount of antimicrobials that finishers at ten farms had been exposed to during their lifetime and independent 
of rearing site. Here, we further develop the approach to provide the LEA estimates for larger population using a systematic computational approach.

In Denmark, all purchases of antimicrobials for use in livestock are registered in the national veterinary medicine register (VetStat) (Stege et al., 2003). Antimicrobials are prescription-only drugs and only sold through veterinarians, pharmacies, or feed mills. The prescribing veterinarian has to reach a diagnosis prior to prescription. Thus, antimicrobials used for prophylactic treatments and as growth promoters are prohibited in Denmark (Anonymous, 2016a). All movements of pigs in Denmark are mandatory to register in the national database for pig movements (PMD). Coupling information on movements of pigs and antimicrobial purchases allows tracing pigs to the farms where they have been and calculate the amount of antimicrobials they were exposed to in the different rearing periods. This is the essence of the LEA approach, which generates data on antimicrobial exposure to batches of pigs with a high resolution both in time (exposure in different rearing periods) and space (usage at different farms). High temporal and spatial resolution is critical in the study of how antimicrobial exposure influences the levels of AMR in livestock animals (Collineau et al., 2017). Henceforth, the LEA estimates may be used for studying the association between antimicrobial exposure and AMR at batch level in population studies and risk assessments where collection of primary data about the antimicrobial usage at farm level is not feasible.

The objective of the presented work was to develop and optimise an algorithm designed to implement the LEA approach presented by Andersen et al (2017) to the majority of slaughter pigs in Denmark. Furthermore, we validate the algorithm using farm data on actual antimicrobial usage. The algorithm was run on Danish farms with a conventional production of slaughter pigs to estimate the proportion of farms for which the algorithm is applicable. 


\section{Materials and methods}

The LEA algorithm estimates the total amount of antimicrobial exposure during the lifetime of a batch of pigs. A batch was defined as a group of pigs sent from the same farm to the same slaughterhouse on the same day. Briefly, the algorithm was run for batches of finisher pigs starting at their date of slaughter. Based on the date of slaughter and identity of the farm with the finisher unit, the algorithm retrospectively estimates at which farm(s) they were reared as piglets and weaners. This tracing process is Step 1 (Estimating the age group of moved pigs) and Step 2 (Production chain trace back) of the algorithm. Subsequently, the antimicrobial exposure per pig per day was estimated for the batches for each of the rearing periods, as described in Step 3 (herd size calculation) and Step 4 (antimicrobial exposure smoothing) of the algorithm (Fig. 1). Finally, the lifetime exposure to antimicrobials was calculated as the sum of antimicrobial exposure in the three rearing periods.

\section{Register data used by the LEA algorithm}

The LEA algorithm used data from three national registers: VetStat, the PMD, and the CHR. The databases are owned by the Danish Veterinary and Food Administration. More information regarding these three databases can be found in in Supplement Material 1.

Data from the CHR were extracted twice - in September 2014 and in March 2015- to account for changes in the farm demographics between the beginning and end of the study period. The number of pigs in each age group (weaners, finishers, and sows) was calculated as the mean of the two data extractions. The PMD data were extracted in April 2015, and data from VetStat were extracted twice - observations from $1^{\text {st }}$ of September 2013 to $30^{\text {th }}$ of April 2015 were extracted in June 2015 and observations for May $1^{\text {st }}$ to July $1^{\text {st }}$ were extracted in October 2015. The two subsets were subsequently merged. 
In this paper, we define a farm as a premise housing pigs according to the CHR.

The algorithm only uses data from pharmacies, accounting for more than $99.9 \%$ of antimicrobials sold for use in pigs in the farms included in the present study (based on calculations from VetStat data).

\section{LEA algorithm}

The LEA algorithm operationally runs in four steps (Fig. 1): 1) Estimating the age group of moved pigs; 2) Production chain trace back; 3) Herd size calculation; 4) Antimicrobial exposure smoothing.

The algorithm deals with three rearing periods of a pig produced for slaughter at approximately 100kg: the piglet period (birth-7 kg), the weaner period (7-30 kg weight) and the finisher period (30-100 kg, approximately).

In this study the LEA algorithm was applied to all Danish pig farms with more than 200 finishers (according to the CHR register data) who sent pigs to slaughter between January $1^{\text {st }}$ and March $201531^{\text {st }}$. For each farm, the batch of pigs that were sent to slaughter closest to the $15^{\text {th }}$ of February 2015 was chosen.

The LEA algorithm was written and run in $\mathrm{R}$ version 3.2.2 ( $\mathrm{R}$ Core Team, 2017). A brief description of each of the four steps follows, and further details are provided in Supplement 2.

\section{Step 1: Estimating the age group of moved pigs}

The PMD contains no information about the age of the pigs moved. In order to calculate the antimicrobial exposure defined by age group, this information must be estimated in order to track the pigs correctly and calculate the number of pigs. The age group was estimated based on:

i) The assumption that a slaughter pig is moved up to three times during its lifetime. The pig is first 
moved from piglet unit to weaner unit at a bodyweight of approximately $7 \mathrm{~kg}$ and then from the weaner unit to a finisher unit at a bodyweight of approximately $30 \mathrm{~kg}$. The movement from a unit to the subsequent unit can happen within the same farm or between farms. The third and final move is from the finisher unit to the slaughterhouse at a bodyweight of approximately 100 $\mathrm{kg}$. The only certain movement is to the slaughterhouse.

ii) The number of pigs moved. There is a limit to the number of pigs of a specific age group a vehicle may transport at once. We assumed that, in the majority of cases, the number of pigs transported would be as close to this limit as possible and consequently that the number of pigs moved would reflect the age group of the moved animals. We do not have an exact estimate for number of pigs moved that would reflect a specific age group. Therefore, we tested different settings (see the section "test of the parameter settings"). To distinguish between movements of sows and movement of finishers, the cut-off $\mathrm{sow}_{\text {sow }}$ wefined as either 40 or 60 pigs per movement. To distinguish between movements of finishers and movement of weaners, the cutoff $_{\text {weaner }}$ for the number of pigs moved was defined as either 250 or 350 pigs per movement. The values for the cut-offs were based on the median number of pigs moved to and from farms with only one age group. Only one set was used in the final algorithm

iii)The information about sending and recipient farms. In the algorithm, a farm could only send and receive pigs of an age group present on the farm according to the CHR. This criterion ensured that when pigs were moved into a farm only having finishers, the pigs would be defined as finishers, irrespective of the number of pigs moved. Furthermore, pigs sent to a slaughterhouse only for finishers, were defined as finishers. Likewise, pigs sent to a slaughterhouse that only slaughtered sows were defined as sows.

\section{Step 2: Production chain trace back}

The algorithm works retrospectively for each batch of pigs by first identifying the farm(s) with the 
weaner unit and then the farm(s) with the piglet unit.

Transfer windows were defined in order to estimate the timeframe at which a pig would progress from the piglet unit to the weaner unit and from the weaner unit to the finisher unit (Fig. S1). The timeframe of the transfer windows were defined using the weight gain statistic for farms with the highest and lowest weight gain. The transfer window was defined as the difference in days between fast (median for $25 \%$ fastest growing pigs) and slow growing pigs (median for the $25 \%$ slowest growing pigs)(Jessen, 2015). The daily weight gain statistic, which describes how fast the pigs are growing in different rearing periods were used to estimate the duration (in number of days) of the rearing period within each rearing unit (piglet, weaner, and finisher unit). The transfer windows were extended to fit irregular movement patterns. Three sets of increases (either $50 \%, 100 \%$, or $250 \%$ of the original transfer window) were assessed. Only one value was used in the final algorithm.

With the date of slaughter as onset, the algorithm retrospectively identified weaner and piglet units where the pigs were housed during their weaner and piglet period, respectively. If the pigs were moved to a new farm when changing rearing periods, the new farm was identified based on the transfer window and the PMD (see step 2 in the Supplementary Material 2).

\section{Step 3: Herd size calculation}

The algorithm estimates the LEA for a batch of pigs. However, pigs within the batch are housed with other pigs, and the purchase of antimicrobials is registered at farm level. Therefore, the exposure for the batch has to be calculated as an average for all pigs in the same age group present at the farm during the rearing period. In the CHR, the number of pigs are reported as the average number of pigs on a normal day of production (Anonymous, 2016b), which is most likely an imprecise estimate of the true number of pigs. Therefore, the LEA algorithm aims at obtaining a better estimate of the average number of pigs at the farm in each of the rearing periods. This is 
calculated based on information found in the CHR, corrected using records of the movements into and out of the farm (see supplement 2).

\section{Step 4: Antimicrobial exposure smoothing}

The LEA algorithm calculates the antimicrobial exposure per batch. This is done for each of the three rearing periods. The estimates for antimicrobial exposure are calculated as a sum of average daily $\mathrm{ADD}_{\mathrm{kg}}$ per pig across the period when the batch was assumed to be present in the rearing unit. The antimicrobial exposure is calculated per class of antimicrobial for 11 different classes, and as a total of all antimicrobials used during each rearing period. These 11 classes of antimicrobials cover all antimicrobial usage in the Danish pig production. For the combination products, the amount of active product was calculated per antimicrobial class and included in the sum of that class.

Antimicrobial exposure is measured in Animal Defined Daily Doses for treatment of one kg $\left(A D D_{k g}\right)$. The $\mathrm{ADD}_{\mathrm{kg}}$ is defined as the average approved dose for the main indication in the particular animal species for treatment of one kilogram pig. The $\mathrm{ADD}_{\mathrm{kg}}$ can be used across age groups, as it is independent of animal bodyweight (Jensen et al., 2011). The antimicrobial exposure is calculated as the sum of $\mathrm{ADD}_{\mathrm{kg}}$ used per day per pig in the batch. Thus, the estimated exposure does not take into account, when the pigs were treated, but merely estimates the amounts of antimicrobial used for the production of a given pig.

In VetStat, antimicrobial purchases for use in sows and piglets are registered in the same age group category. Previous studies have shown that antimicrobial exposure of the sow will affect the AMR pattern in the piglet (Callens et al., 2015; Mathew et al., 2005). Therefore, we use the antimicrobial purchase for use in sows and piglets as an estimate for the antimicrobial exposure of piglets, thus including both the direct and indirect selection pressure on the piglet microbiota.

No information about the duration of treatment, number of pigs treated, or exact dates of treatment 
are available in the VetStat data. In general, the veterinarian is allowed to prescribe antimicrobials that are expected to be needed until next planned visit. Therefore, it was assumed that antimicrobials would be used between the purchase date of a product and the following purchase date of a product with the same antimicrobial class and dispersing form (parenteral or oral). We call this our "smoothing method". Details about the smoothing and calculation of antimicrobial exposure can be found in supplementary material 2 .

\section{Test of the parameter settings}

Only batches of pigs where the LEA algorithm could identify at least one weaner unit and one piglet unit were included in the further analyses. The tracing of pigs back to the weaner and piglet units depended on the setting of the transfer windows and the identification of the age group of the pigs moved in the PMD. The LEA algorithm was run 12 times with different settings for the transfer window, cut-off weaner $_{\text {and cut-off }}$ sow to evaluate the effectiveness of the algorithm to trace the pigs back to weaner and piglet units. The parameter settings optimizing the number of batches where both weaner and piglet units could be identified were chosen for the final LEA algorithm.

\section{Adjustments to the number of pigs calculated}

The LEA algorithm does not accurately calculate the number of pigs present for all of the farms. The number of finishers and weaners registered in the CHR is used as an alternative to the LEA estimate of the number of pigs present in the following circumstances:

i) If the number of weaners and finishers is calculated to be zero or below zero.

ii) If the absolute difference between the calculated number of pigs and the number of pigs registered in the CHR for the age group, divided by the number of pigs registered in the CHR, is calculated to be above 0.9 .

iii) If the absolute value for the movement balance ratio is calculated to be above 1 . The movement 
balance ratio is calculated as the sum of pigs produced at the farm and the number of pigs sent out, subtracted by the number of pigs received, and subsequently divided by the total number of pigs registered at the farm.

All above of the above are results of missing information of movements of pigs into or out of the farm causing an underestimation or overestimation of the number of pigs present at the farm; or because the weight gain parameters did not fit the specific farm.

\section{Validation of the smoothing method}

The validity of our smoothing method was assessed by comparing the estimated value of exposure to actual farm registrations of antimicrobial usage.

We obtained data from 7 finisher units, 4 weaner units, and 4 piglet units, including one farm with all three units (an integrated farm). The finisher and weaner units received pigs from the weaner and piglet units, respectively. The following information was available in the data registered on the farms: the farm number (CHR-number), age group of treated animals, date of start of treatment, date of end of treatment, product name, and amount of product used in the duration of the treatment.

For the period, where farm registrations were available, up to 15 dates of slaughter was chosen at random using the "sample"-function in R (R Core Team, 2017). These dates were used to set the periods for which the antimicrobial exposure should be calculated. The periods were set to be 75 days (number of days in the finisher period, see supplementary material 2) prior to the date of slaughter. This was done to be able to run the smoothing part of the LEA algorithm.

For the piglet and weaner units, we chose up to 30 slaughter dates at random for the finisher units that received pigs from the piglet and weaner units. These were sampled among slaughter dates ensuring that there was farm data available. Hereafter, with use of the sampled slaughter dates and number of days in each period (30 and 58 days, defined in Supplement Material 2) the dates for the 
piglet and weaner periods were defined. This was done to be able to run the smoothing part of the LEA algorithm for the defined dates.

The smoothing step of the LEA algorithm (included in Step 4) was run for the defined periods for the finisher, weaner, and piglet units to obtain estimates of antimicrobial exposure. The farm records were used to calculate the amount of antimicrobials used in each period. Both antimicrobial estimates were calculated in $\mathrm{ADD}_{\mathrm{kg}}$ without taking into account the number of pigs. This was done to be able to only validate the smoothing method. The two antimicrobial estimates (smoothed and farm data) were compared in scatterplots and the spearman correlation was calculated between the two estimates for the piglet, weaner, and finisher units, respectively.

\section{Lifetime exposure to antimicrobials and movement patterns}

The output of the LEA algorithm was used to describe the movement patterns and, the patterns of antimicrobial exposure in the Danish pig population.

The distribution of antimicrobial exposure was visualized using boxplots. To make the visual presentation of the output of the LEA algorithm comparable to official reports of the antimicrobial usage in Denmark (Danish Veterinary and Food Administration, 2017) the antimicrobial exposure was standardized to $\mathrm{ADD} / 100$ pigs/day. This was calculated as the $\mathrm{ADD}_{\mathrm{kg}}$ divided by the standard weight at treatment (sows: $200 \mathrm{~kg}$, weaners: $15 \mathrm{~kg}$, finishers: $50 \mathrm{~kg}$ ) and divided by the number of days in each period.

All analyses were done in R (R Core Team, 2017) using RStudio (RStudio team, 2016).

\section{Results}

\section{Test of the parameter settings}

The settings for the transfer window and the settings for the cut-offs affected the number of farms 
that were traceable. The optimal parameter settings were as follows: the transfer window was increased by $250 \%$ compared to the original transfer window (Fig. S1), the cut-off ${ }_{\text {weaner }}$ was set to 250 pigs, and the cut-off ${ }_{\text {sow }}$ was set to 60 pigs (Fig. 2). The percentage of the farms, where an associated weaner unit could be identified was slightly higher $(94.33 \%)$ for the chosen setting of $250 / 250 / 60$, than the setting of 250/250/40 (94.28 \%). The settings for the transfer window affected the number of instances where no weaner units could be identified. The settings for the cut-off weaner $_{\text {in }}$ affected the number of batches of pigs that were traceable to the piglet unit.

\section{Validation of smoothing estimation}

Figure 3 shows the comparison between the LEA smoothed purchase data and actual farm usage data used to validate the smoothing method. The spearman correlations were piglet period: $\rho=0.9$, weaner period: $\rho=0.6$, finisher period: $\rho=0.6$, all $p$-values $<0.0001$. The correlations were higher for the weaner and finisher periods, when pleuromutilins were not included in the calculations, $\rho=0.8$ and $p$-values $<0.0001$ for both. The correlations were based on 101 observations from the 7 finisher units, 51 observations from the 4 weaner units, and 99 observations for the 4 piglet units.

\section{Lifetime exposure to antimicrobials and movement patterns}

There were 3,954 conventional farms, with more than 200 finisher pigs registered in CHR, sending pigs to slaughter between January $1^{\text {st }}$ and March $31^{\text {st }} 2015$. Hereof, $928(23 \%)$ were excluded because they were not traceable by the LEA-algorithm.

In total, we estimated the LEA for batches of pigs originating from finisher units at 3,026 farms (77 $\%)$, hereafter referred to as the traceable batches of pigs. The batches of pigs had spent their weaner period at 1,991 different farms and their piglet period at 1,524 different farms.

For 962 of the farms with finisher units for the traceable batches, the number of finishers registered in the CHR was used instead of the calculated number of finishers. The calculated number of 
weaners was exchanged for the number of weaners registered in the CHR for 412 of the 1,991 farms with weaner units.

Figure 4 illustrates the different lifetime movement patterns for $80 \%$ of the traceable batches of pigs together with the percentage of batches showing a particular movement pattern. The remaining $20 \%$ of the batches had very different movement patterns, each describing $<1 \%$ of the batches. The movement of the majority slaughter pigs can be described by 9 different patterns (Fig. 4). The distribution of exposure (measured as average amount of ADD per 100 pigs per day in the batch) to different antimicrobial classes in each rearing period can be seen in Figure 5. The lifetime exposure to antimicrobials can be seen in Figure 6.

\section{Discussion}

There were 3,954 farms with conventional production of finishers in the register data used in this study. This paper presents an implementation of the LEA approach on the routinely registered data about antimicrobial purchase and movement of pigs between farms in a computational algorithm that enabled estimation of the antimicrobial exposure from birth to slaughter at batch level for $80 \%$ of Danish pig farms with finisher units. The LEA algorithm facilitates estimation of the antimicrobial exposure for a batch of pigs slaughtered on a given date and is based on statistics regarding production parameters and the use of register data. The LEA algorithm therefore enables retrospective estimation of the antimicrobial exposure during different rearing periods and in total in a large population. As shown in this study, $80 \%$ of finisher pigs, which could be traces back, are moved to a new farm at some point in time. Studies have shown that antimicrobials consumed in the early stages of a pig's life affect the level of antimicrobial resistance found in later stages (Dunlop et al., 1998; Rosengren et al., 2007; Varga et al., 2009). It is therefore important to consider all rearing periods when assessing the effect of antimicrobial exposure on AMR in 
slaughter pigs.

In the LEA algorithm, there are assumptions about age groups of moved pigs, number of days spent in each rearing unit, and the duration of treatment. These assumptions were necessary because we lack specific information at farm level regarding these parameters. Therefore, the estimated exposure to antimicrobials in the different batches is surrounded with some uncertainty. The assumption with highest influence on the traceability of the batches concerned the increase of the transfer windows (Fig. 2). The cut-off values used to define the age group in PMD could be rendered unnecessary by implementing the age group in the PMD.

In the data on antimicrobial purchases from VetStat, no information is available on the duration of treatment. Therefore, we assumed that the amount of antimicrobials of a given antimicrobial class and dispensing form (parenteral or oral) will be used until the next date when a product of same antimicrobial class and dispensing form was purchased. This assumption was validated using data from farm records on antimicrobial usage. We found generally a good correlation between the smoothed estimate and the farm data. This indicates that the smoothing method gives a sufficiently good estimation of the actual exposure level and duration of treatments. This is recommended by Collineau et al (2016) to include in studies of the relationship between AMR and antimicrobial exposure, which is the intended use of the LEA algorithm. However, differences between the smoothed and the actual farm data were observed, most profound for pleuromutilins (Fig. 3). Whether this difference is caused by the algorithm or that pleuromutilins sometimes are purchased for use in one age group but used in another is unknown. The difference was particularly observed in the batches from the integrated farm (results not shown). The LEA algorithm cannot take into account whether antimicrobial products are purchased for use in one age group, but in reality used in another age group, because only purchasing information is available in the register data. Our results show that in some farms sometimes antimicrobials are used for another age group than the 
age group it was prescribed and purchased for. For instance, in Figure 3, according to the LEA algorithm pleuromutilins are often used in finisher units. However, in several of these farms the real farm data showed a zero ADD value for pleuromutilins. In the weaner units, the pattern is opposite. Several of the weaner units using pleuromutilins according to the real farm data had no use according to the LEA algorithm. In $90 \%$ of the finisher units purchasing but not using pleuromutilins in finishers, the pleuromutilins were used in a weaner unit at the same farm. Other antimicrobial classes were also purchased for use in one age group but used in another. However, the pattern was most pronounced for pleuromutilins. Considering the estimation of lifetime exposure, the discrepancy between which age group the antimicrobials has been prescribed to and the actual use will be partly excluded from the estimate of the LEA algorithm, because lifetime exposure ignores the actual age when the pigs have been exposed to the antimicrobial.

Another assumption in the LEA algorithm was the number of days in each rearing period. This assumption was based on the median weight gain per rearing period of Danish pig farms (Jessen, 2015). These values were fixed, meaning that the same values were used for all farms. This was due to the lack of information of farm specific weight gains, which could be obtain through productivity data. Applying productivity data at farm level would increase the validity of the LEA algorithm. However, this estimate cannot be obtained from all farms and we therefore did not endeavour further to integrate it into the LEA algorithm at this stage. Furthermore, there is only a three-nine day difference in time between the median growing pigs and the fastest or slowest growing pig depending on the rearing period (Fig. S1). Therefore, we believe that using fixed values for number of days in each rearing period do not add a substantially error.

We found that the LEA algorithm could be used to trace back to the farm of origin for $77 \%$ of Danish pig farms with a conventional production of finishers and with more than 200 finishers registered in CHR. The excluded farms were found to be significantly smaller than the included 
ones. It is the nature of the algorithm that batches of pigs from integrated farms will not be excluded as they do not require data from the movement database to be assigned a piglet and weaner unit. It was estimated that $20 \%$ of the excluded farms were excluded due to lack of registrations in the PMD. The remaining cases were excluded due to production systems not covered by the algorithm. These production systems could have a shorter or longer nursing time as well as a lower or higher daily weight gain. Although registers on farm demographics and the movement of pigs in Denmark do exist, they are neither perfect nor complete, as reflected in this study.

Using the data on antimicrobial purchases available from VetStat poses further challenges. One challenge is that antimicrobials purchased for use in piglets will be registered for use in sows. Thus, the LEA algorithm estimates the antimicrobial use for production of slaughter pigs including the antimicrobial use for the sows in the piglet unit. However, the selection pressure on the environment of the piglet (including the sow) is also of relevance for the occurrence of AMR in the piglet (Callens et al., 2015; Mathew et al., 2005). Nevertheless, the predictive value would presumably be better if the antimicrobial use in the sow and the piglet could be separated.

The LEA algorithm was used to describe the pattern of antimicrobial exposure in Danish batches of slaughter pigs (Fig. 5 and 6). Raw values of the sum of average daily amount of antimicrobial exposure showed that the pigs are exposed to larger amounts of antimicrobials in the finisher period than in the weaner period (results not shown). However, standardizing the values to ADD/100 pigs/day showed that the weaners are exposed to a higher dose per day than the finishers (Fig. 5) due to lower bodyweight and a lower number of days in the weaner period. The algorithm estimates only pigs slaughtered in Denmark. Consequently, the antimicrobial consumption of weaners exported to be fattened abroad are not part the antimicrobial estimates in the weaner units. 


\section{Conclusions}

We have developed an algorithm (LEA), which based purely on register data, production parameter statistics, and relatively few assumptions estimates the lifetime antimicrobial exposure for a batch of pigs, regardless of whether the pigs remained at the same farm or were moved to other farms during their lifetime. The LEA algorithm works for the majority of pig farms in Denmark. This gives us the opportunity to study the association between antimicrobial exposure and AMR at batch level in large population studies and to perform risk assessments at national level.

\section{Acknowledgements}

We would like to thank Mette Fertner and Nana Dupont for help calculating the $\mathrm{ADD}_{\mathrm{kg}}$ from the VetStat data, and discussions about how to calculate the LEA. Furthermore, we would like to thank Amanda Kruse, Gitte Blach Nielsen and Leonardo de Knegt for a valuable discussion leading to the assumption used in the LEA algorithm. Finally, we would like to thank Mette Ingvordsen, Lisbeth Harm Nielsen and Jan Dahl for feedback on the initial LEA algorithm. This study was funded by the Danish Food and Veterinary Administration. The funding party had no influence on the design of the study. 


\section{References}

Andersen, V.D., DE Knegt, L. V, Munk, P., Jensen, M.S., Agers $\varnothing$, Y., Aarestrup, F.M., Vigre, H., 2017. The association between measurements of antimicrobial use and resistance in the faeces microbiota of finisher batches. Epidemiol. Infect. 1-11. doi:10.1017/S0950268817001285

Anonymous, 2016a. Order (DK) 1533/2016 Regarding the Veterinarian's use, dispense and prescription of medicines for animals [in Danish], of December 12, 2016. Denmark.

Anonymous, 2016b. Order (DK) 1408/2016 Regarding registration of herds in CHR [in Danish], of November 29, 2016.

Callens, B., Faes, C., Maes, D., Catry, B., Boyen, F., Francoys, D., de Jong, E., Haesebrouck, F., Dewulf, J., 2015. Presence of antimicrobial resistance and antimicrobial use in sows are risk factors for antimicrobial resistance in their offspring. Microb. Drug Resist. 21, 50-8. doi:10.1089/mdr.2014.0037

Collineau, L., Belloc, C., Stärk, K.D.C., Hémonic, A., Postma, M., Dewulf, J., Chauvin, C., 2017. Guidance on the Selection of Appropriate Indicators for Quantification of Antimicrobial Usage in Humans and Animals. Zoonoses Public Health 64, 165-184. doi:10.1111/zph.12298

Danish Veterinary and Food Administration, 2017. yellow card initiative [WWW Document]. URL http://www.foedevarestyrelsen.dk/english/Animal/AnimalHealth/Pages/The-Yellow-CardInitiative-on-Antibiotics.aspx (accessed 1.7.17).

Dunlop, R.H., McEwen, S.A., Meek, A.H., Clarke, R.C., Black, W.D., Friendship, R.M., 1998. Associations among antimicrobial drug treatments and antimicrobial resistance of fecal Escherichia coli of swine on 34 farrow-to-finish farms in Ontario, Canada. Prev. Vet. Med. 34, 283-305.

Jensen, V.F., Emborg, H.D., Aarestrup, F.M., 2011. Indications and patterns of therapeutic use of 
antimicrobial agents in the Danish pig production from 2002 to 2008. J. Vet. Pharmacol. Ther. 35, 33-46. doi:10.1111/j.1365-2885.2011.01291.x

Jessen, O., 2015. National average for productivity in the pig production 2014 [in Danish].

Mathew, A.G., Garner, K.N., Ebner, P.D., Saxton, A.M., Clift, R.E., Liamthong, S., 2005. Effects of antibiotic use in sows on resistance of E. coli and Salmonella enterica Typhimurium in their offspring. Foodborne Pathog. Dis. 2, 212-20. doi:10.1089/fpd.2005.2.212

R Core Team, 2017. R: A Language and Environment for Statistical Computing.

Rosengren, L.B., Waldner, C.L., Reid-Smith, R.J., Dowling, P.M., Harding, J.C.S., 2007. Associations between feed and water antimicrobial use in farrow-to-finish swine herds and antimicrobial resistance of fecal Escherichia coli from grow-finish pigs. Microb. Drug Resist. 13, 261-69. doi:10.1089/mdr.2007.781

RStudio team, 2016. RStudio: Integrated Development Environment for R.

Stege, H., Bager, F., Jacobsen, E., Thougaard, A., 2003. VETSTAT-the Danish system for surveillance of the veterinary use of drugs for production animals. Prev. Vet. Med. 57, 105115. doi:10.1016/S0167-5877(02)00233-7

Varga, C., Rajić, A., McFall, M.E., Reid-Smith, R.J., Deckert, A.E., Checkley, S.L., McEwen, S. a, 2009. Associations between reported on-farm antimicrobial use practices and observed antimicrobial resistance in generic fecal Escherichia coli isolated from Alberta finishing swine farms. Prev. Vet. Med. 88, 185-92. doi:10.1016/j.prevetmed.2008.10.002

Wickham, H., 2009. ggplot2: Elegant Graphics for Data Analysis. Springer-Verlag New York. 


\section{Figure legends}

Figure 1: Overview of the four steps in the LEA algorithm. The figure shows the register data and assumptions used in each step. PMD: Database of Pig Movements, CHR: Central Husbandry Register, PMDr: revised Database of Pig Movements (after estimating the age group of moved pigs). The weight gain statistics were obtained from Danish production statistics (Jessen, 2015).

Figure 2: Performance of the traceability of the LEA algorithm with different parameter settings. The percentage of the traceable batches of pigs is shown as a function of the parameter settings:



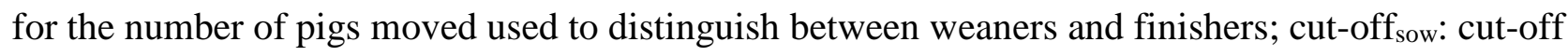
for the number of pigs moved used to distinguish between sows and finishers. *tw: transfer window.

Figure 3: Validation of the smoothing method. Scatterplot of the correlation between smoothed antimicrobial exposures estimated from the LEA-algorithm $\left(\mathrm{LEA} \mathrm{ADD}_{\mathrm{kg}}\right)$ and data on antimicrobial usage from farm records (farm $\mathrm{ADD}_{\mathrm{kg}}$ ). The line indicates the perfect linear association between the two measures. Ext.: extended.

Figure 4: Lifetime movement patterns for the traceable batches of pigs, and the percentage of the batches with the specific movement pattern. "Traceable" refer to that the LEA algorithm could assign at least one weaner and one piglet unit to the batch. The figure illustrates approximately 80 $\%$ of the traceable batches of pigs. The remaining $20 \%$ had very diverse movement patterns and each of these patterns represented less than $1 \%$ of the farms. One horisontal bar is one physical farm. An arrow indicate a movement to a new farm or a movement to the slaughterhouse.

Figure 5: Antimicrobial exposure in the three rearing periods, described for each antimicrobial type. The antimicrobial exposure was standardized to ADD/100 pigs/day. This was calculated as the 
$\mathrm{ADD}_{\mathrm{kg}}$ divided by the standard weight at treatment (sows: $200 \mathrm{~kg}$, weaners: $15 \mathrm{~kg}$, finishers: $50 \mathrm{~kg}$ ) and divided by the number of days in each rearing period. Two observations of antimicrobial exposure in the weaner period were removed due to high numbers (ADD/100 pigs/day of 1 ) that masked the overall distributions of the remaining observations. Lower and upper hinges describe the $25^{\text {th }}$ and $75^{\text {th }}$ percentiles and the middle hinge describes the median. The lower and upper whiskers extend from the lower and upper hinges to $+/-1.5$ times the distance between the $25^{\text {th }}$ and $75^{\text {th }}$ percentiles. Values below the lower whiskers or above the higher whiskers are considered outliers, and are plotted as dots (Wickham, 2009).

Figure 6: Lifetime exposure to 11 different antimicrobial classes. Five observations of lifetime exposure were removed due to high numbers $\left(\mathrm{ADD}_{\mathrm{kg}}\right.$ above 1000) that masked the overall distributions of the remaining observations. Lower and upper hinges describe the $25^{\text {th }}$ and $75^{\text {th }}$ percentiles and the middle hinge describes the median. The lower and upper whiskers extend from the lower and upper hinges to $+/-1.5$ times the distance between the $25^{\text {th }}$ and $75^{\text {th }}$ percentiles. Values below the lower whiskers or above the higher whiskers are considered outliers, and are plotted as dots (Wickham, 2009). 


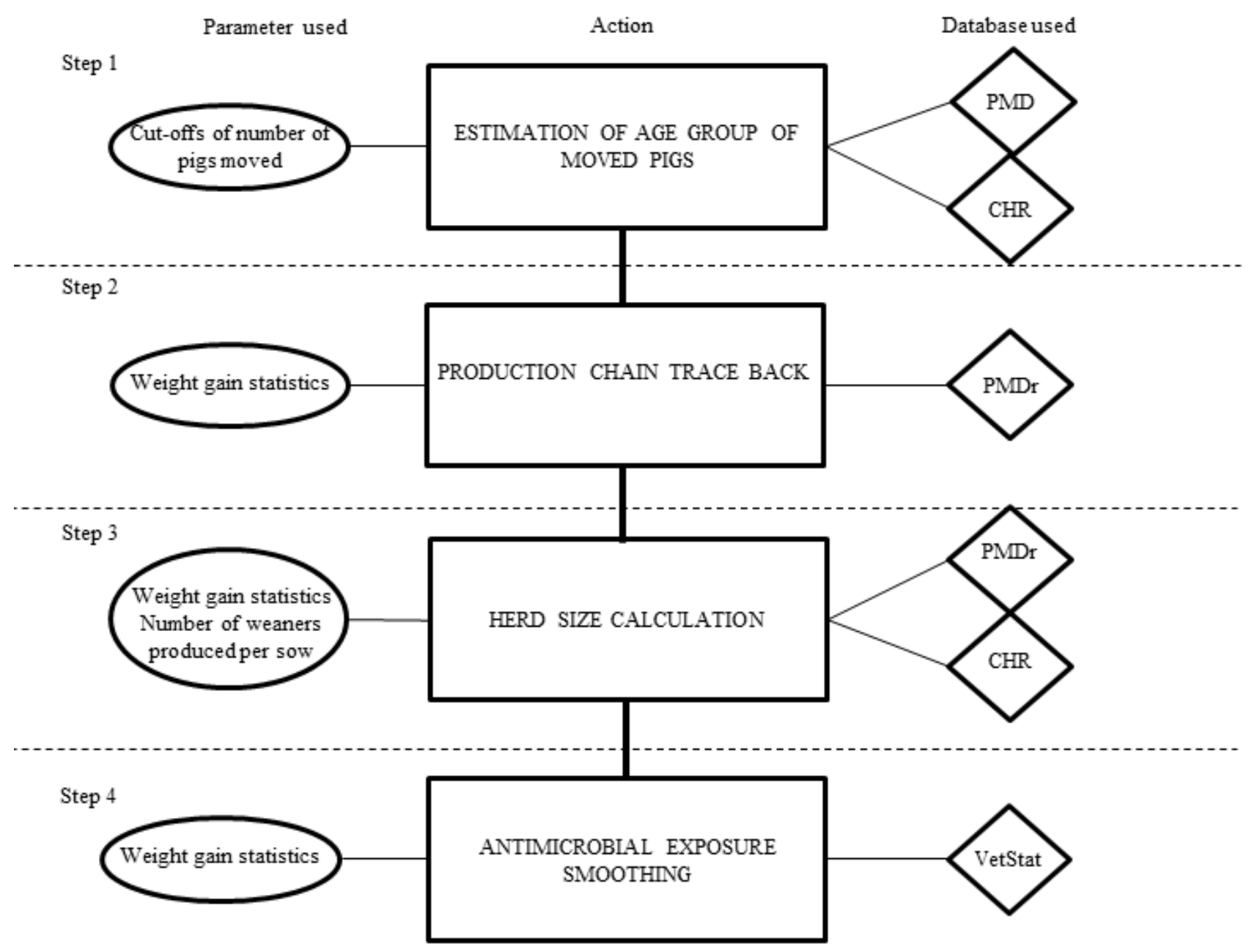




\section{at least one $\bullet$ weaner unit $\bullet$ sow unit}
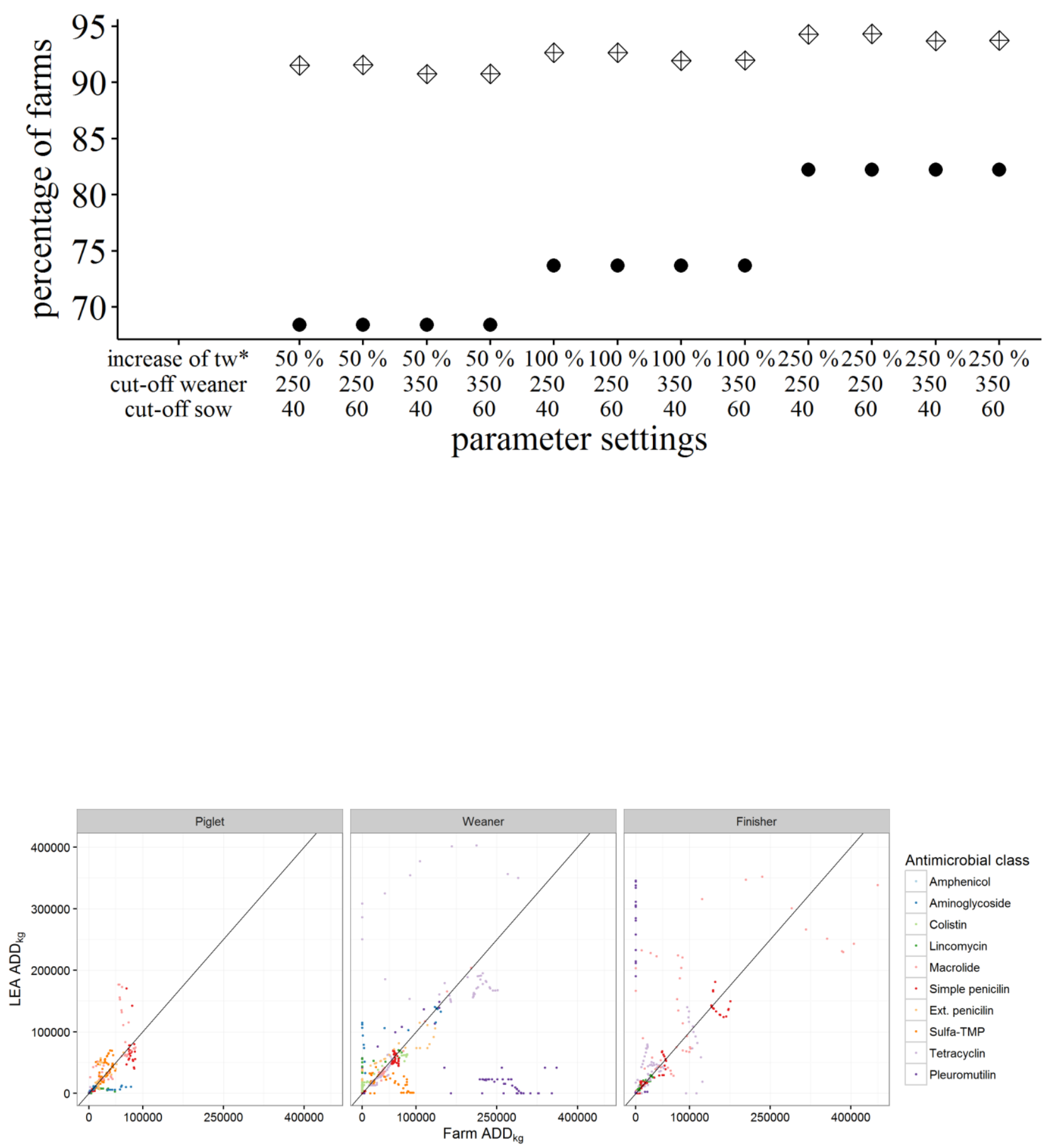

Antimicrobial class

Amphenicol

Aminoglycoside

Colistin

Lincomycin

Macrolide

Simple penicilin

Ext. penicilin

Sulfa-TMP

Tetracyclin

Pleuromutilin 


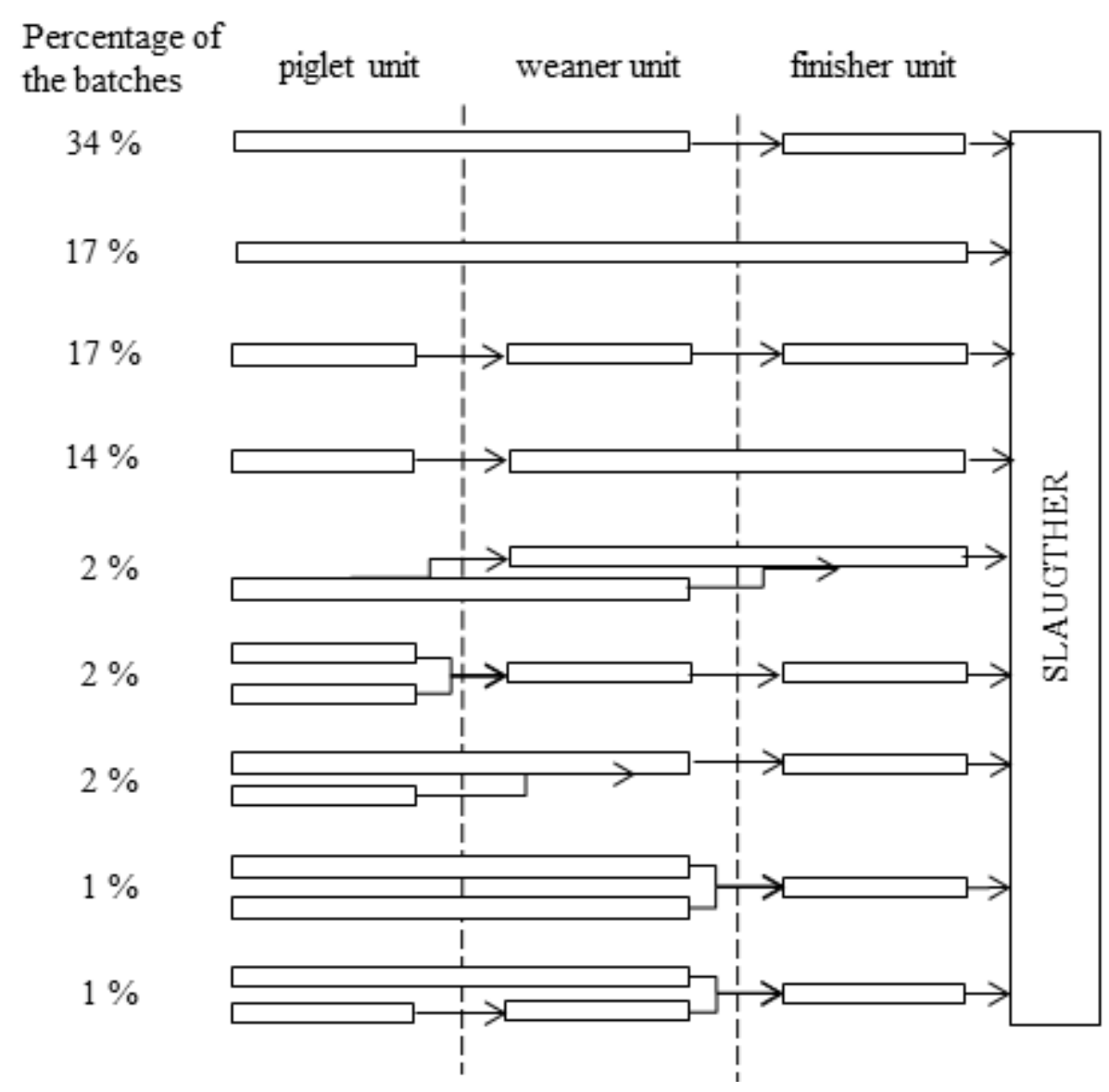




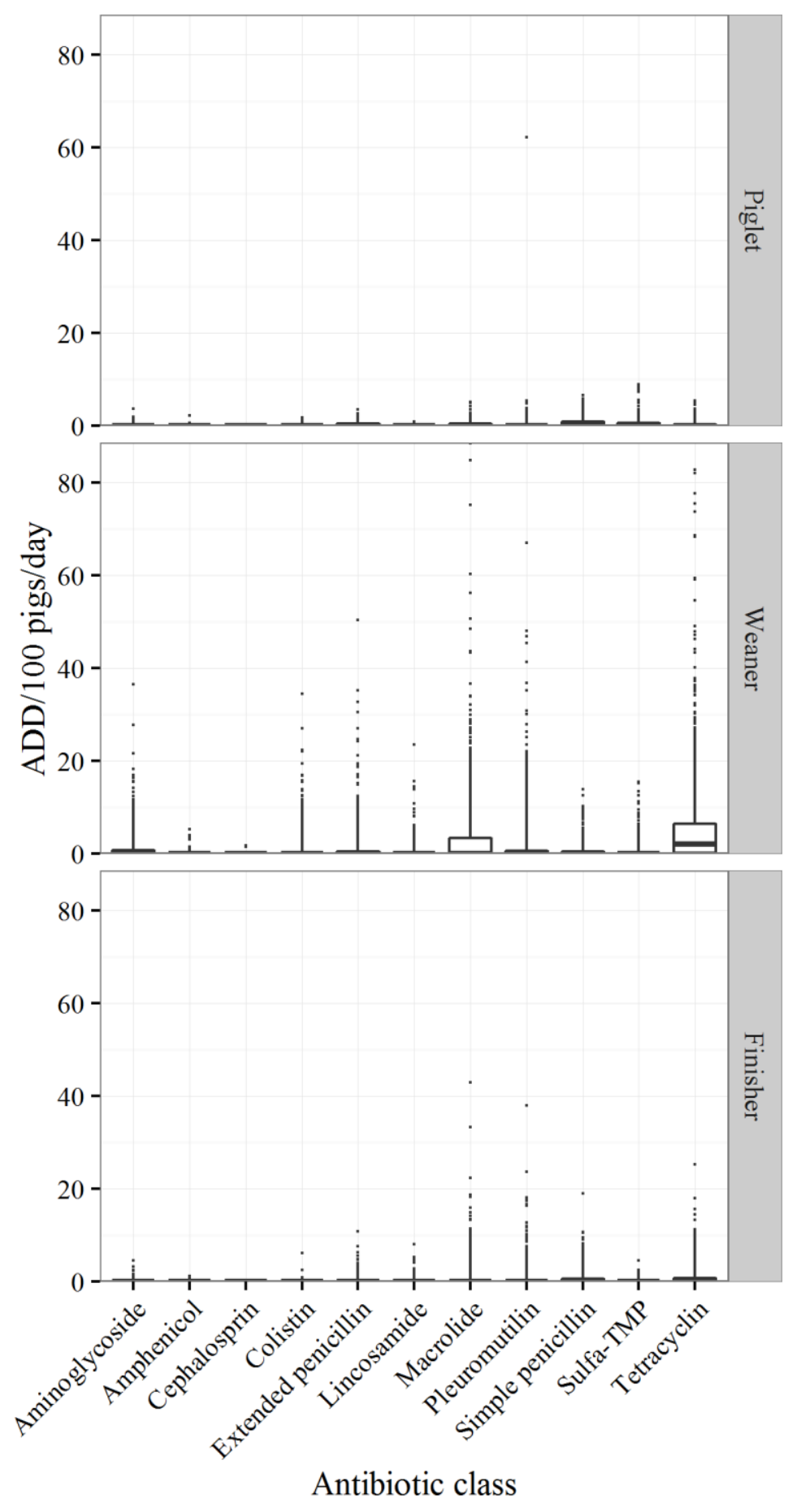




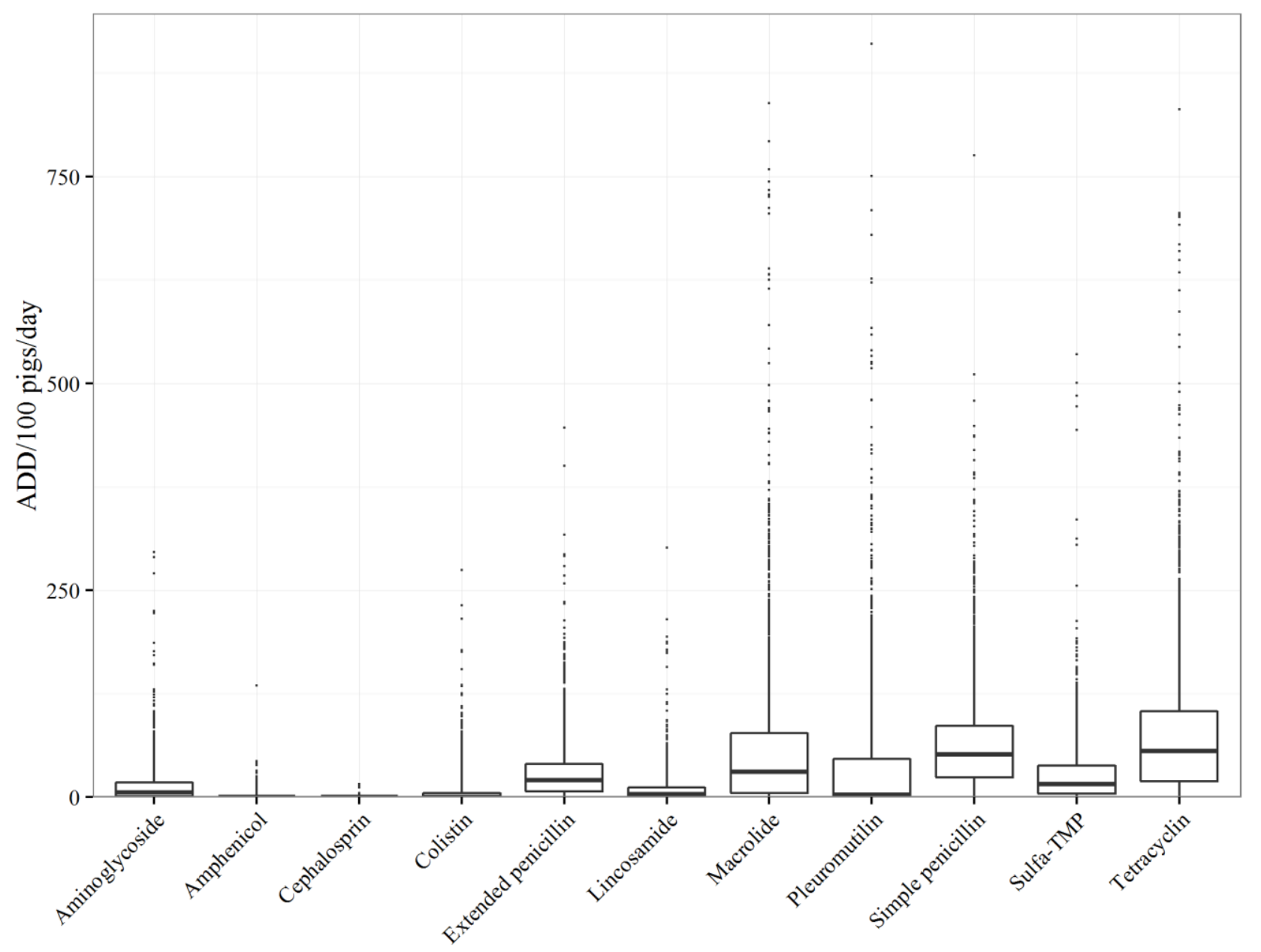

Antibiotic class 